\title{
MicroRNAs in neural cell development and brain diseases
}

\author{
FENG Wei \& FENG Yue* \\ Department of Pharmacology, School of Medicine, Emory University, Atlanta, GA 30322, USA
}

Received September 12, 2011; accepted September 22, 2011

\begin{abstract}
MicroRNAs play important roles in post-transcriptional regulation of gene expression by inhibiting protein translation and/or promoting mRNA degradation. Importantly, biogenesis of microRNAs displays specific temporal and spatial profiles in distinct cell and tissue types and hence affects a broad spectrum of biological functions in normal cell growth and tumor development. Recent discoveries have revealed sophisticated mechanisms that control microRNA production and homeostasis in response to developmental and extracellular signals. Moreover, a link between dysregulation of microRNAs and human brain disorders has become increasingly evident. In this review, we focus on recent advances in understanding the regulation of microRNA biogenesis and function in neuronal and glial development in the mammalian brain, and dysregulation of the microRNA pathway in neurodevelopmental and neurodegenerative diseases.
\end{abstract}

microRNAs, neuronal development, synaptic plasticity, oligodendroglial differentiation, brain tumor, neurodegenerative disorders

Citation: Feng W, Feng Y. MicroRNAs in neural cell development and brain diseases. Sci China Life Sci, 2011, 54: 1103-1112, doi: 10.1007/s11427-011$4249-8$

\section{Molecular mechanisms that control biogene- sis of microRNAs}

MicroRNAs are short 18-25 nucleotide small non-coding RNA molecules that function to silence gene expression via sophisticated post-transcriptional regulation [1]. In mammals, the primary precursors of microRNAs (pri-microRNAs) are transcribed by RNA polymerase II (for details of microRNA biogenesis, see recent reviews [2,3]) and cleaved by the nuclear RNase III endonuclease, Drosha, along with its regulatory subunit DGCR8. As a result, characteristic 70-100 nucleotide stem-loop precursor microRNAs are generated (pre-microRNAs) in the nucleus, sometimes even derived from introns after RNA splicing [4-6]. Pre-microRNAs are exported to the cytoplasm by Exportin5 and further processed by the RNase III, Dicer, to

*Corresponding author (email: yfeng@emory.edu) produce mature microRNAs of short duplexes from the terminal loop. Besides this canonical pathway for microRNA biogenesis, emerging evidence indicates the existence of atypical mechanisms for transcription and processing of virally encoded pri-microRNAs that are closely related to transfer RNA (tRNA) biogenesis [7], which in turn converge on the DICER-dependent pathway to generate mature viral microRNAs. Whether cellular orthologues for components in this atypical pathway exist or this is a viral-specific mechanism remains unknown. Moreover, the biogenesis and stability of specific microRNAs can be modulated by selective RNA-binding proteins (RBPs) that are not core components of the microRNA machinery. In particular, numerous RBPs were recently found to modulate microRNA processing. For example, RBP LIN28 suppresses the biogenesis of let-7 microRNA by binding to the terminal loop of pri-let-7, thereby blocking Drosha cleavage [8]. Interestingly, in cells lacking LIN28, the KH-type splicing regulatory protein (KHSRP, also known as KSRP) recog- 
nizes the terminal loop of let-7 and promotes its maturation by facilitating its association with Drosha in the nucleus or with Dicer in the cytoplasm [9]. In contrast, another canonical splicing regulator, heteronuclear ribonucleoprotein A1 (hnRNPA1), binds to the same sequence in the pri-let-7 terminal loop to repress Drosha-mediated processing [9]. In fact, many RBPs classically known for regulating premRNA splicing are now found to play crucial roles in modulating microRNA biogenesis.

The mature microRNA duplexes are incorporated into the RNA-induced silencing complex (RISC) consisting of Dicer, TRBP, and Ago proteins, in which one or both strands of a microRNA are presented to cognate binding sites, also called microRNA recognition elements (MREs), in the protein-coding mRNA(s) to form an RNA hybrid via imperfect complementary base pairing. MREs are often located in the $3^{\prime}$ untranslated regions (UTRs) of the target mRNAs [10], although clear examples also demonstrate the capability for microRNAs to target the open reading frame of the target mRNA [11-13]. A single microRNA has the potential to target hundreds of distinct mRNA molecules to suppress expression of the encoded protein. On the other hand, multiple microRNAs are predicted to act on the same mRNA and most likely synergistically function to suppress expression of the target. Overall, more than $50 \%$ of human mRNAs are predicted to be targets of microRNAs [14]. Multiple molecular mechanisms have been reported that underlie microRNA-mediated gene silencing, including block of cap-dependent translation initiation, shortening of the polyA tail length, and degradation of the target mRNAs [15]. Besides targeting mRNAs, the most recent discoveries have revealed a new model for microRNA function, in which specific microRNAs, represented by miR-709 and miR-107, can directly target microRNA biogenesis posttranscriptionally [16,17], demonstrating a functional hierarchy within the microRNA pathway. Moreover, RBPs play a role in activation or relief of microRNA repression. For example, HuR, an AU-rich element-binding protein, relieves the miR-122-mediated repression of CAT-1 mRNA by interfering with microRNA binding to the $3^{\prime}$-UTR of target mRNAs [18]. In contrast, repression of c-Myc mRNA by the let-7 microRNA is enhanced by the HuR binding to adjacent AREs [19]. This suggests that regulation of microRNA-mediated repression by RBPs is probably a widespread phenomenon.

Despite the ubiquitous presence and essential roles of the microRNA pathway in all cell types examined [20], expression of microRNAs in response to external signals is highly dynamic at both spatial and temporal levels, which is an apparently important mechanism that governs cell growth and development [1]. Interestingly, microRNAs are particularly abundant in the brain. At least $60 \%$ of known microRNA species are detected in the adult brain [21] and many are drastically regulated during embryonic brain development [22]. Upon neural lineage specification, a sub- class of microRNAs, represented by miR-9, miR-124, and miR-128, are specifically expressed in neurons and play pivotal roles in neuronal development and synaptic plasticity. In contrast, distinct microRNA species are preferentially expressed in glial lineages, which control normal glia cell proliferation and differentiation [23-25]. Specifically, miR-219 and miR-338 are crucial for the development of oligodendroglia, the cell type responsible for myelination of neuronal axons to enable proper conductivity in the brain [23]. Besides cell type-specificity, particular microRNAs demonstrate unique patterns of regional and subcellular localization in the brain. For example, miR-218, miR-221, miR-222, miR-26a, miR-128a/b, miR-138, and let-7c are preferentially enriched in the hippocampus, while miR-195, miR-497, and miR-30b are found to be enriched in the cerebellum [21]. This regional specificity in microRNA expression reflects the different cell composition within various brain regions. miR-134 and miR-26a are relatively enriched in neuronal dendrites in which local protein synthesis underlies synaptic plasticity, the foundation for learning and memory [26,27]. Consistent with the idea that microRNAs may be involved in modulating local protein synthesis, miR-200c, miR-339 and miR-332 are present in biochemical preparations that are enriched in synapses [28]. Examples of microRNAs in specific cell lineages and subcellular compartments are illustrated in Figure 1.

\section{Essential roles of microRNA pathways in dis- tinct neural cell types}

Because conventional deletion of the Dicer gene arrests microRNA processing and results in early embryonic lethality [29], conditional knockout of Dicer, which is required for microRNA biogenesis, has been used extensively to examine the collective roles of microRNAs in specific tissues and cell types in mice. Elimination of Dicer in neural stem cells causes profound defects in embryonic brain development and lethality [30,31]. Apoptosis and failures in developing specialized neuronal arborizations are common detrimental effects in distinct neuronal populations as a result of losing DICER function. In specific neuronal cell types, the loss of Dicer in mature dopaminergic neurons leads to a progressive loss of midbrain dopaminergic neurons and markedly reduced locomotion, which is reminiscent of human patients with Parkinson's disease [32]. Ablation of Dicer function in Purkinje neurons leads to Purkinje cell death, followed by cerebellar degeneration and the development of ataxia [33]. Moreover, conditionally inactivate Dicer in forebrain and hippocampal neurons results in changes in dendrite morphology, spine length, apoptosis, microcephaly, ataxia, and lethality by 3 weeks after birth [34]. The importance of microRNA biogenesis is further supported by the fact that haploinsufficiency of the microRNA processer, Dgcr8, also leads to altered plasticity 


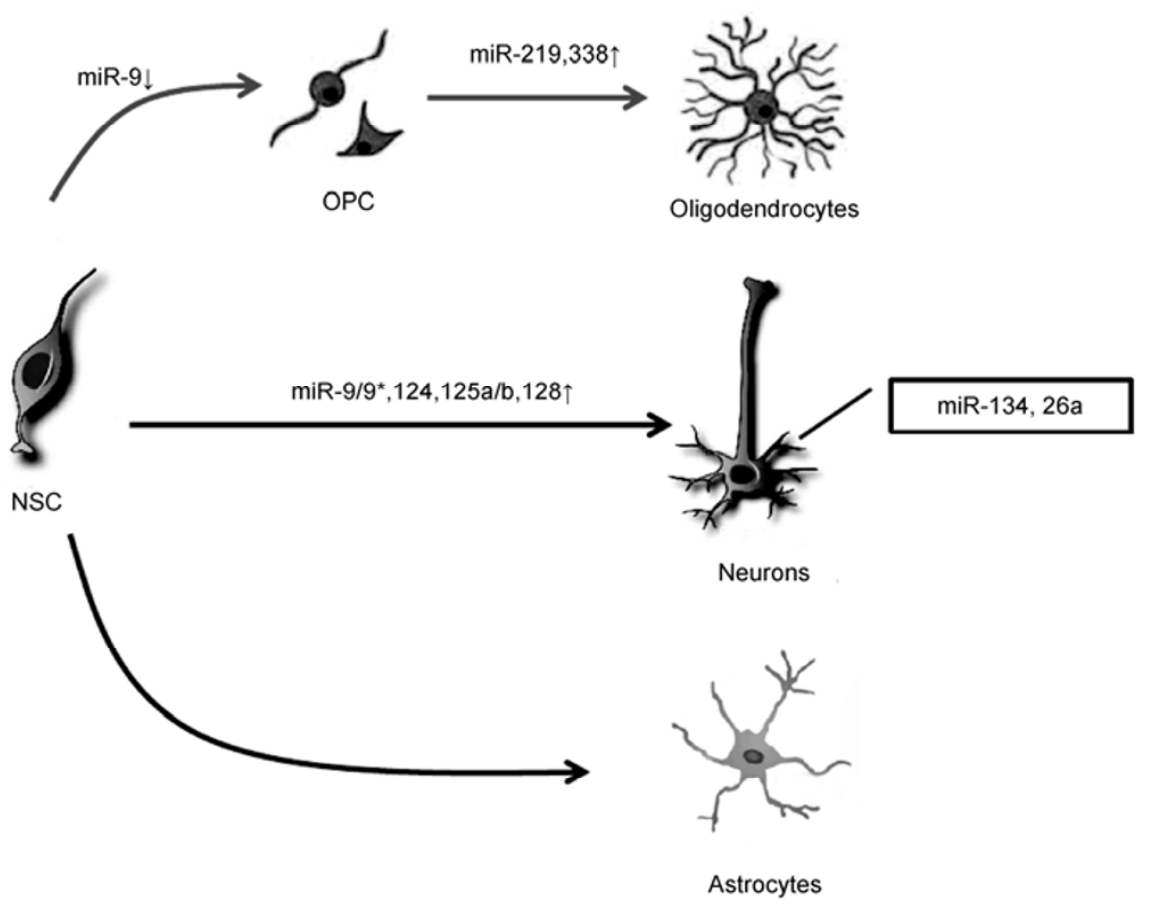

Figure 1 MicroRNA expression in specific cell lineages.

and cognitive dysfunction [35-37].

Besides brain neurons, functional requirements of microRNA pathways are also demonstrated by conditional knockout of Dicer in specialized neural cells in the retina, cochlea, and olfactory bulb. In addition, loss of Dicer in distinct glial cell populations also severely affects the development and function of the nervous system, because of the crucial roles of glia in supporting and modulating neuronal function [38]. Specifically, Dicer deficiency in the oligodendroglia lineage and Schwann cells causes failures in myelination of the central and peripheral nervous system (CNS and PNS) respectively [24,25,39-41]. Interestingly, mice that loose Dicer in astrocytic glia do not show severe defects until the age of five weeks, characterized by progressive ataxia, cerebellar degeneration and seizures, consistent with the critical role of astroglia in neurotransmitter abundance and modulation of synaptic function [42]. All these observations clearly demonstrated the profound global function of microRNA pathways in the nervous system. The functional impairment of the nervous system by conditional elimination of microRNA biogenesis in various neural cell types is summarized in Table 1 .

\section{Specific microRNAs in neuronal development and synaptic plasticity}

Several specific microRNAs have been found to have important effects on neuronal development via regulation of mRNA targets. For example, neuron-specific miR-124 promotes neuronal differentiation by directly targeting PTB, which encodes a global repressor for alternative pre-mRNA splicing in non-neuronal cells [43]. The miR124-dependent silencing of PTB in neural stem cells is an important mechanism that permits neuronal lineage establishment and neuronal differentiation. Antagonizing miR-124 results in sustained PTB expression and suppresses neuronal-specific alternative splicing, and severely attenuates neuronal differentiation [43]. In addition, miR-9* and miR-124 inhibit neural progenitor cell specific BAF53a and BAF45a respectively, reducing proliferation and inducing differentiation [44]. Moreover, expression of miR-9/9* and miR-124 (miR-9/9*-124) in human fibroblasts is sufficient to induce their conversion into neurons [45]. These studies indicate that neuronal-specific microRNAs have instructive roles in neural fate determination.

In addition to neural lineage development, proper synaptic strength and connectivity are a crucial prerequisite for the function of the brain. In mature neurons that are connected in sophisticated synaptic circuitry, a number of mature microRNA species are found in the dendrites and synapses where active protein synthesis occurs locally to govern synaptic plasticity and cognitive ability [28,46], suggesting a function of microRNAs in synaptic mRNA translation. Two profiling studies have identified dendriticenriched microRNAs by either laser capture microdissection of dendrites isolated from primary rat hippocampal neurons [27] or biochemical isolation of synaptosomes, a biological fraction enriched for synaptic terminals [47]. The presence of functional components of the microRNA pathway in- 
Table 1 Functional impairment by conditional elimination of microRNA biogenesis

\begin{tabular}{|c|c|c|c|}
\hline Specific cell types & Specific Cre & Phenotypes & Ref \\
\hline Purkinje cells & Pcp2-Cre-Dicer1 & Cell death, cerebellar degeneration, ataxia & [33] \\
\hline Dopaminergic neurons & DAT-Cre-Dicer1 & Cell loss, reduced locomotion & [32] \\
\hline Dopaminoceptive neurons & DR-1-Cre-Dicer1 & $\begin{array}{l}\text { Ataxia, front and hind limb clasping, reduced brain size, and } \\
\text { smaller neurons but not cell death }\end{array}$ & [48] \\
\hline Spinal motor neurons & VAChT-Cre-Dicer1 & $\begin{array}{l}\text { Sclerosis, aberrant end plate architecture, myofiber atrophy, } \\
\text { and spinal muscular atrophy }\end{array}$ & [49] \\
\hline $\begin{array}{l}\text { Neural stem and progenitor } \\
\text { cells in cortex }\end{array}$ & Emx1-Cre-Dicer1 & $\begin{array}{l}\text { Apoptosis, impairs neuronal differentiation, thinner cortical } \\
\text { wall and death before P } 30\end{array}$ & {$[30,31,50]$} \\
\hline $\begin{array}{l}\text { Neural stem and progenitor } \\
\text { cells in brain }\end{array}$ & Nestin-Cre-Dicer1 & $\begin{array}{l}\text { Thinner cortical wall (E18.5), reduced number of late-born } \\
\text { neurons and abnormal migration; very few embryos, no sur- } \\
\text { viving newborns }\end{array}$ & [31] \\
\hline $\begin{array}{l}\text { Neurons of cortex and hip- } \\
\text { pocampus }\end{array}$ & CaMKII $\alpha$-Cre-Dicer & $\begin{array}{l}\text { Dendritic branch elaboration reduces, dendritic spine length } \\
\text { increases, increased cortical apoptosis, smaller cortex, mi- } \\
\text { crocephaly, tremors, ataxia, and early postnatal death before } \\
\text { P21 }\end{array}$ & {$[34,51]$} \\
\hline Adult forebrain neurons & CaMK-CreERT2-Dicer1 & $\begin{array}{l}\text { Tamoxifen begins } 8-10 \text { weeks, a cumulative neuronal cells } \\
\text { loss after } 14 \text { weeks of tamoxifen, improved learning and } \\
\text { memory, increases posttetanic potentiation, elongated filo- } \\
\text { podia-like shaped dendritic spines }\end{array}$ & [52] \\
\hline Retina & Chx10-Cre-Dicer1 & $\begin{array}{l}\text { Retina cells disorganization and degeneration, morphological } \\
\text { defects, ERG responses decreases, inability to respond to } \\
\text { light }\end{array}$ & [53] \\
\hline Retinal progenitor cells & $\alpha$ Pax6-Cre-Dicer1 & Increases early-born cell types, fail to generate late-born cells & [54] \\
\hline Retinal progenitor cells & DKK3-Cre-Dicer1 & $\begin{array}{l}\text { Massive death of retinal progenitor cells, abnormal cell dif- } \\
\text { ferentiation }\end{array}$ & [55] \\
\hline Inner ear hair cells & Pou4f3-Cre-Dicer1 & Deaf, with no response to auditory stimuli & [56] \\
\hline Olfactory progenitor cells & Foxg1-Cre-Dicer1 & $\begin{array}{l}\text { Die in utero, have small eyes and forebrains, and develop } \\
\text { small snouts }\end{array}$ & [57] \\
\hline Mature olfactory neurons & OMP-Cre-Dicer1 & Viable, show normal olfactory-related functions & [57] \\
\hline OPCs and Oligodendrocytes & Olig2-Cre-Dicer1 & Demyelinating phenotypes begin P10, but disappear at $\mathrm{P} 60$ & [24] \\
\hline OPCs and Oligodendrocytes & Olig1-Cre-Dicer1 & $\begin{array}{l}\text { Demyelinating phenotype (severe tremor, ataxia, unmyelin- } \\
\text { ated axons), die week } 3\end{array}$ & [25] \\
\hline OPCs and Oligodendrocytes & Oligo1-Cre-Dicer1 & $\begin{array}{l}\text { Oligodendrogenesis suppressed, astrogliogenesis is absent, } \\
\text { die immediately after birth. }\end{array}$ & [58] \\
\hline OPCs & CNP-Cre-Dicer1 & $\begin{array}{l}\text { Demyelinating (P9-10, develop notable tremor), peripheral } \\
\text { neuropathy, die week } 4\end{array}$ & [24] \\
\hline OPCs & CNP-Cre-Dicer1 & Oligodendrocytes reduces, die before adulthood & [59] \\
\hline Mature oligodendrocytes & PLP-CreERT-Dicer1 & $\begin{array}{l}\text { CNS impairment (ataxia, paralysis, kyphosis, and early } \\
\text { death); progressively severe hind limb ataxia until paralysis } \\
\text { and kyphosis }\end{array}$ & [39] \\
\hline Schwann cells & Dhh-Cre-Dicer1 & $\begin{array}{l}\text { Arrest at immature stages, demyelinating (discoordination, } \\
\text { tremor, and ataxia), aggravated as older }\end{array}$ & [40] \\
\hline Schwann cells & P0::Cre-Dicer1 & $\begin{array}{l}\text { Arrest at immature stages, demyelinating (clenching and } \\
\text { hindlimb), aggravated as older }\end{array}$ & [41] \\
\hline Astrocytes & mGFAP-Cre-Dicer1 & $\begin{array}{l}\text { Normal before week } 5 \text {, then progressive ataxia, cerebellar } \\
\text { degeneration, seizures, uncontrollable movements, and } \\
\text { premature death by postnatal week } 9-10\end{array}$ & [42] \\
\hline All tissues & $\operatorname{Dgcr}^{+/-}$ & $\begin{array}{l}\text { Homozygous mice die before birth, heterozygous reduce ex- } \\
\text { citatory synaptic transmission and display cognitive deficits }\end{array}$ & [35-37] \\
\hline
\end{tabular}

dendrites, including Dicer and Argonaute, as well as pre-microRNAs, further implicate the regulation of microRNA biogenesis [46], in response to neuronal activity changes.

Indeed, neuronal activity has a profound impact on all aspects of microRNA biogenesis and function. At the transcriptional level, membrane depolarization or application of the neurotrophin, BDNF, activate transcription of miR-132 by the well-known activity-regulated transcription factor CREB [60], while, neuronal activity activates miR-134 transcription by the transcription factor MEF2 [26]. In contrast, the dendritically enriched miR-138 is specifically expressed in brain neurons, while its precursor pre-miR-138 is ubiquitously expressed, suggesting specific post-transcriptional processing of miR-138 in neurons [61], although neuronal specific transacting factors that enable processing of pre-miR-138 are yet to be identified. Furthermore, emerging evidence has revealed rapid regulation of numerous microRNAs upon neuronal activation under specific neurotransmitter receptor-mediated synaptic signaling, most likely via regulating the abundance of the microRNA processing machinery [46]. Besides microRNA abundance, it is important to point out that an expanding number of reports demonstrate interplay between microRNAs and RBPs that act on the same mRNA targets to modulate microRNA activity [62]. The well characterized RBP, HuR, is translocat- 
ed to the cytoplasm upon stress, which relieves cationic amino acid transporter 1 (CATI) mRNA from miR-122mediated repression [18]. In addition, phosphorylation of the fragile $\mathrm{X}$ mental retardation protein, FMRP, was recently shown to promote the formation of an AGO2-miR125 a inhibitory complex on PSD-95 mRNA, which is reversed upon mGluR-triggered dephosphorylation of FMRP [63].

It is clearly demonstrated that activity-regulated microRNAs can control neuronal development and function. In a well characterized example, miR-132 induces formation of dendritic spines and increases the occurrence of miniature EPSCs by repressing expression of p250GAP, a GTPase activating protein that regulates the Rac1/PAK1 pathway [64]. In addition, miR-134 regulates dendrite growth by targeting RNA-binding protein, Pumilio2 [26], and decreases dendritic spine size by repressing LIMK1, a kinase that promotes actin polymerization [65]. In contrast, miR-138 induces spine shrinkage by targeting depalmitoylation enzyme, APT1, and by activation of the RhoA-ROCK pathway [47].

\section{MicroRNAs in oligodendroglial differentia- tion and myelin formation}

Oligodendroglia in the CNS produce multilamellar myelin sheaths wrapping around neuronal axons, which are essential for axonal protection and saltatory conduction of action potentials. Oligodendroglia progenitor cells are specified from neural stem/progenitor cells, which proliferate and differentiate, and ultimately migrate to interact with axons where they become mature oligodendrocytes to form myelin sheaths. Abnormal formation and/or maintenance of myelin sheaths impairs nerve conduction and leads to progressive axonal degeneration, which contributes to the etiology of several neurological disorders, including multiple sclerosis and schizophrenia. A rich and fast growing literature has demonstrated that many intrinsic and extrinsic regulators control myelinating cell differentiation in a spatiotemporally specific manner. MicroRNAs, as novel post-transcriptional regulators, act cooperatively with developmental signals to control oligodendrocyte differentiation and myelin formation. In vivo elimination of Dicer1, specifically in oligodendroglia, by Olig1, Olig2, or CNP promoter-directed expression of the Cre-recombinase causes severe dysmyelination and motor behavior deficits, characterized by tremors, ataxia and paralysis [24,25,58,59], demonstrating essential roles of microRNA pathways in oligodendrogliadependent CNS myelination. In fact, OL-specific Dicer mutants suffer from oxidative damage, inflammatory astrocytosis and microgliosis in the brain, which eventually leads to neuronal degeneration and shorter lifespans [39]. Recent microarray profiling studies identified specific microRNAs that are rigorously regulated during oligodendroglial differ- entiation [66], supporting the idea that developmentally programmed microRNA expression governs oligodendroglial lineage development. Interestingly, the neuronal specific miR-9 is down-regulated during the transition from oligodendroglial progenitor cells that still maintain a certain plural potency to mature oligodendrocytes [23]. This suggests that miR-9 may play opposing roles in neuronal and glial lineage specification. Regarding functional microRNA species in oligodendroglia, several studies collectively demonstrate that miR-219 is a major microRNA necessary and sufficient to promote oligodendrocyte terminal maturation [24,25]. miR-138 and miR-338 also belong to the same class that advances oligodendrocyte differentiation [25]. Mechanistically, these aforementioned microRNAs directly target and silence transcription factors that suppress oligodendroglial differentiation, including ZFP238, FoxJ3, Sox3, and Hes5 [24,25], as well as PDGF receptor alpha, which mediates PDGF signaling to maintain oligodendroglial progenitors [67]. Later on in mature oligodendrocytes, miR-219 is believed to target the elongation of very long chain fatty acids protein 7 (ELOVL7) to maintain lipids and redox homeostasis, which are necessary for supporting axonal integrity as well as for the formation of compact myelin. Considering the pivotal role of miR-138 in controlling neuronal dendritic spine morphogenesis [47], whether miR-138 acts through shared mRNA targets in neurons and oligodendroglia or alternatively targets distinct downstream pathways, perhaps with the help of neuronal and/or glial specific factors, still remains elusive.

\section{MicroRNAs in brain tumor development}

Primary brain tumors, though relatively rare, are the cause of a disproportionate level of morbidity and mortality and have been the subject of increasingly intensive research over the past two decades. Glioma, the most common primary tumor of the adult CNS, develops from fundamental genetic alterations that cause the formation of a tumor stem cell population that divides without restriction to normal physiological biochemical signaling. Gliomas are classified based on the different glial cells types present, including ependymomas (ependymal cells), astrocytomas (astrocytes), oligodendrogliomas (oligodendrocytes) and glioblastoma multiforme (GBM), the most frequent and malignant primary brain tumor [68]. In contrast, medulloblastoma (MB) is the most common brain malignancy in children that arises from altered development of cerebellar progenitor cells [69]. MicroRNAs certainly play critical roles in cancer initiation, progression, and some of them could be considered as clinical biomarkers for cancer diagnosis, prognosis and prediction of therapeutic response [70]. Specifically, expression profiling studies have revealed extensive alteration of microRNAs in primary glioblastoma, suggesting that microRNAs may represent a new class of genes involved in 
Table 2 MicroRNAs function as oncomicroRNAs or anti-oncomicroRNAs in glioma and medulloblastoma biology

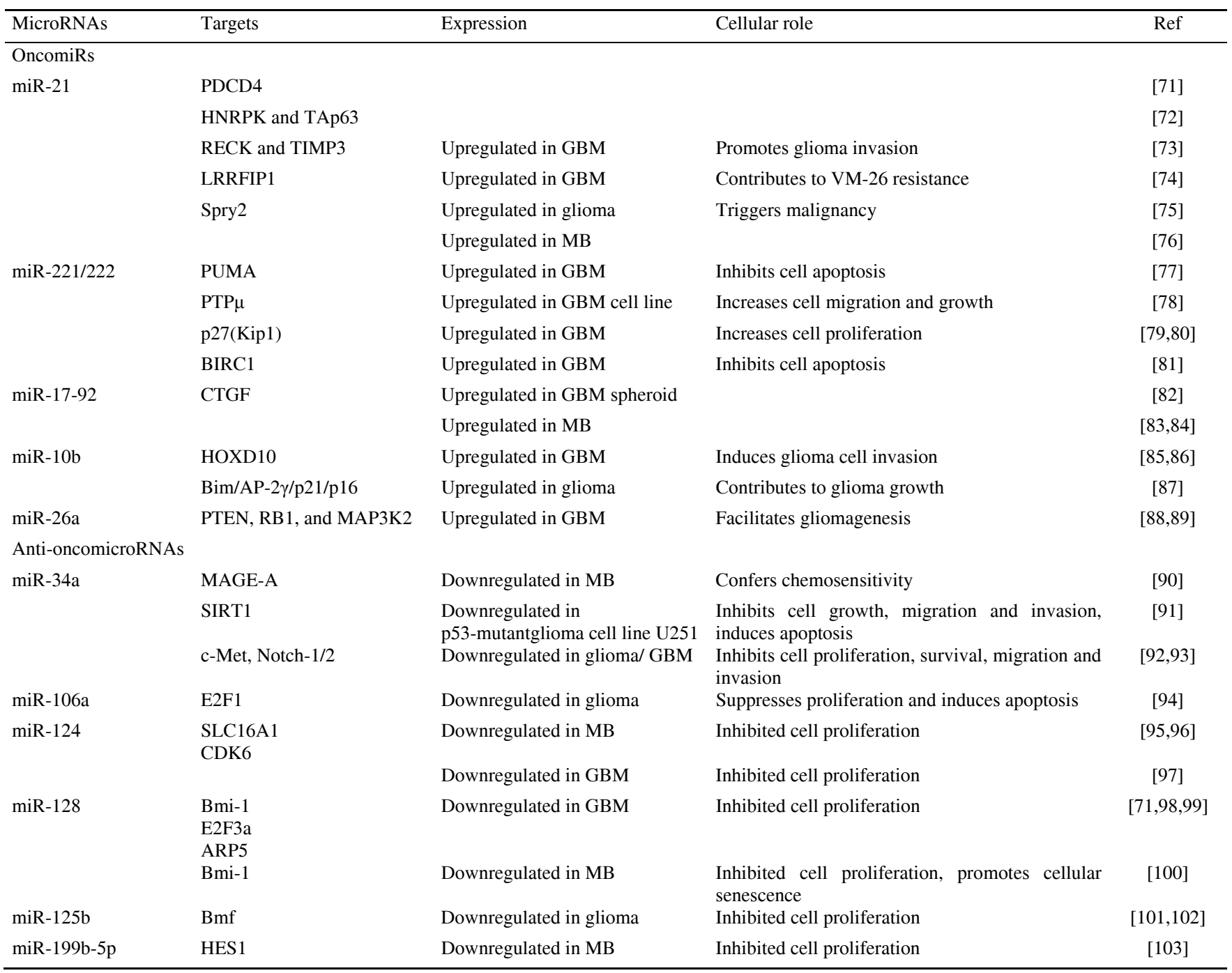

oncogenesis [104]. The dysregulated microRNAs in GBM could be characterized as oncomiRs or anti-oncomiRs through post-transcriptionally regulating oncogenes or anti-oncogenes, respectively (Table 2). In GBM, the dysregulation is characterized by the strong up-regulation of miR-221, accompanied by a much broader spectrum of down-regulated miRs, including miR-128, miR-181a, miR-181b, and miR-181c [104]. Interestingly, dysregulation of microRNAs in MBs is characterized by a somewhat overlapping microRNA population [105], especially the predominant down-regulation of a class of neural microRNAs, suggesting a tumor growth-inhibitory function of these microRNAs. It is worth pointing out that besides serving as a biomarker for tumor development, some microRNAs appear to be drug targets. For example, in glioma, knockdown of miR-21 increased apoptotic activity, and sensitized cells to cytotoxic tumor therapy [106-109]. In medulloblastoma, suppression of miR-21 impedes cell migration [76]. These pioneer studies support alternative promising strategies to treat brain tumors.

\section{MicroRNAs in neurodevelopmental and neurodegenerative disorders}

A rapidly growing literature has demonstrated that altered neuronal plasticity and morphology, as seen in neurodevelopmental disorders, may result from disruption of a common post-translational process that is under tight control by microRNAs [110]. Several intellectual disability syndromes, such as Fragile X syndrome, Rett syndrome, and Down's syndrome, have been linked to the microRNA pathway [110]. Fragile X syndrome, characterized by intellectual disability and autistic features, is caused by loss of function of the fragile $\mathrm{X}$ mental retardation protein (FMRP). FMRP is involved in the microRNA pathway through interaction with Dicer and Ago1 [111] and association with specific microRNAs [112]. Specifically, miR-125b and miR-132 
have been shown to associate with FMRP in the mouse brain and they exert opposing effects on dendritic spine morphology and synaptic plasticity. Loss of FMRP ameliorates the microRNAs effects on spine morphology [112]. In addition, FMRP indirectly regulates miR-124 levels by interaction with the Dicer1-Ago1-complex in Drosophila [113], suggesting FMRP may maintain proper levels of many microRNAs in neuronal development.

Rett syndrome is an X-linked neuro-developmental disorder caused by mutations in the transcriptional co-repressor methyl CpG-binding protein (MeCP2). Upregulation or downregulation of MeCP2 both lead to Rett-like syndrome in mouse, suggesting maintenance of MeCP2 levels in a narrow range is essential for normal brain development and function [114]. miR-132, a brain-enriched microRNA, has been shown to inhibit MeCP2 translation [115]. More interestingly, loss of MeCP2 leads to down-regulation of miR-132 by indirect mechanisms [115]. This negative feedback loop provides a mechanism for tight homeostatic control of MeCP2 expression [115]. Moreover, alterations in microRNA profiles in MeCP2-knockout mouse models reveal that up-regulated microRNAs are responsible for the decreases of BDNF and Irak1 [116,117], which are crucial players for normal brain development.

Parkinson's disease (PD) is characterized by the progressive neurodegeneration of dopaminergic neurons (DNs) in the substantia nigra, leading to tremors, rigidity, and bradykinesia [118]. Conditional knockout of Dicer in dopaminergic neurons results in Parkinson's disease-like phenotypes with or without cell loss [32,43], suggesting microRNAs regulate homeostatic states in dopaminergic neurons. Consistent with this view, miR-133b is significantly downregulated in midbrain tissue from patients with Parkinson's disease [32]. Further studies revealed that miR-133b regulates the maturation and function of midbrain DNs within a negative feedback circuit that includes the paired-like homeodomain transcription factor, Pitx3 [32]. In addition, miR-7 reduces alpha-synuclein protein, the major component of Lewy bodies in sporadic PD, through binding the 3'-UTR of alpha-synuclein mRNA and protecting cells against oxidative stress $[119,120]$. Furthermore, the reduction of miR-7 in the MPTP-induced PD model further supports the role of miR-7 in controlling alpha-synuclein levels [119], which is critical for the neurodegenerative process in PD.

Finally, emerging evidence also reveals the involvement of microRNA malfunction in Alzheimer's disease (AD), a progressive neurological degenerative disorder caused by the accumulation of plaques formed of short $\beta$-amyloid (A $\beta$ ) peptides. These peptides arise from the proteolytic cleavage of the $\beta$-amyloid precursor protein (APP), by a $\beta$-secretase known as the $\beta$-site APP-cleaving enzyme (BACE), and $\gamma$-secretase. BACE1, a rate-limiting step enzyme for $A \beta$ peptide production, is thought to be an important risk factor for sporadic AD. Importantly, a number of microRNAs exhibit abnormal expression levels in postmortem AD brain tissues compared to matched normal controls [121], and many of the altered microRNAs in AD have been shown to regulate BACE1 [122-124]. Moreover, APP levels are also known to be under the regulation of microRNAs [125-128].

\section{Concluding remarks and perspectives}

The pivotal importance of microRNAs in normal development and function of the brain has been clearly demonstrated. The most recent discoveries begin to elucidate an integrated picture regarding the function of specific microRNA species in distinct neural cell lineages, in response to different stimulation cues, as well as with respect to dysregulated microRNAs in various neurological diseases and brain tumors. The changes of microRNA expression patterns can serve as informative biomarkers that indicate the functional status of a normal brain, as well as disease progression and prognosis in brain tumors, neurodevelopmental disorders, and neurodegenerative diseases. Given the crucial function of microRNAs in governing normal brain function and the marked dysregulation of microRNAs in brain diseases, the question of whether microRNA function can be manipulated to obtain therapeutic benefits is an intriguing possibility currently under rigorous investigation. To achieve this goal, understanding the molecular mechanisms that regulate microRNA biogenesis and activity, at epigenetic, transcriptional and post-transcriptional levels, especially by co-operation with selective RBPs in response to developmental and synaptic signals, is an important question that needs to be delineated by future studies. In addition, given the large number of mRNAs predicted to be regulated by microRNAs, a practical strategy that fully integrates identification and validation of microRNA target sites and biological changes of functional mRNA targets by the biological and disease signatures of microRNAs is urgently needed; this is apparently the next challenge. Manipulation of one microRNA might simultaneously restore the level of a large network of disease-related target genes, which seems to be a promising strategy of treatment.

1 Bushati N, Cohen S M. microRNA functions. Annu Rev Cell Dev Biol, 2007, 23: 175-205

2 Kim V N, Han J, Siomi M C. Biogenesis of small RNAs in animals. Nat Rev Mol Cell Biol, 2009, 10: 126-139

3 Flynt A S, Lai E C. Biological principles of microRNA-mediated regulation: shared themes amid diversity. Nat Rev Genet, 2008, 9: $831-842$

4 Ruby J G, Jan C H, Bartel D P. Intronic microRNA precursors that bypass Drosha processing. Nature, 2007, 448: 83-86

5 Berezikov E, Chung W J, Willis J, et al. Mammalian mirtron genes. Mol Cell, 2007, 28: 328-336

6 Okamura K, Hagen J W, Duan H, et al. The mirtron pathway generates microRNA-class regulatory RNAs in Drosophila. Cell, 2007, 
130: $89-100$

7 Bogerd H P, Karnowski H W, Cai X, et al. A mammalian herpesvirus uses noncanonical expression and processing mechanisms to generate viral MicroRNAs. Mol Cell, 2010, 37: 135-142

8 Viswanathan S R, Daley G Q, Gregory R I. Selective blockade of microRNA processing by Lin28. Science, 2008, 320: 97-100

9 Michlewski G, Caceres J F. Antagonistic role of hnRNP A1 and KSRP in the regulation of let-7a biogenesis. Nat Struct Mol Biol, 2010, 17: 1011-1018

10 Brennecke J, Stark A, Russell R B, et al. Principles of microRNA-target recognition. PLoS Biol, 2005, 3: e85

11 Shen W F, Hu Y L, Uttarwar L, et al. MicroRNA-126 regulates HOXA9 by binding to the homeobox. Mol Cell Biol, 2008, 28: 4609-4619

12 Forman J J, Legesse-Miller A, Coller H A. A search for conserved sequences in coding regions reveals that the let-7 microRNA targets Dicer within its coding sequence. Proc Natl Acad Sci USA, 2008, 105: 14879-14884

13 Duursma A M, Kedde M, Schrier M, et al. miR-148 targets human DNMT3b protein coding region. RNA, 2008, 14: 872-877

14 Miranda K C, Huynh T, Tay Y, et al. A pattern-based method for the identification of MicroRNA binding sites and their corresponding heteroduplexes. Cell, 2006, 126: 1203-1217

15 Eulalio A, Huntzinger E, Izaurralde E. Getting to the root of miRNA-mediated gene silencing. Cell, 2008, 132: 9-14

16 Chen P S, Su J L, Cha S T, et al. miR-107 promotes tumor progression by targeting the let-7 microRNA in mice and humans. J Clin Invest, 2011, 121: 3442-3455

17 Tang R, Li L, Zhu D, et al. Mouse miRNA-709 directly regulates miRNA-15a/16-1 biogenesis at the posttranscriptional level in the nucleus: evidence for a microRNA hierarchy system. Cell Res, 2011, in press

18 Bhattacharyya S N, Habermacher R, Martine U, et al. Relief of microRNA-mediated translational repression in human cells subjected to stress. Cell, 2006, 125: 1111-1124

19 Kim H H, Kuwano Y, Srikantan S, et al. HuR recruits let-7/RISC to repress c-Myc expression. Genes Dev, 2009, 23: 1743-1748

20 Lagos-Quintana M, Rauhut R, Yalcin A, et al. Identification of tissue-specific microRNAs from mouse. Curr Biol, 2002, 12: 735-739

21 Bak M, Silahtaroglu A, Moller M, et al. microRNA expression in the adult mouse central nervous system. RNA, 2008, 14: 432-444

22 Miska E A, Alvarez-Saavedra E, Townsend M, et al. Microarray analysis of microRNA expression in the developing mammalian brain. Genome Biol, 2004, 5: R68

23 Lau P, Verrier J D, Nielsen J A, et al. Identification of dynamically regulated microRNA and mRNA networks in developing oligodendrocytes. J Neurosci, 2008, 28: 11720-11730

24 Dugas J C, Cuellar T L, Scholze A, et al. Dicer1 and miR-219 Are required for normal oligodendrocyte differentiation and myelination. Neuron, 2010, 65: 597-611

25 Zhao X, He X, Han X, et al. MicroRNA-mediated control of oligodendrocyte differentiation. Neuron, 2010, 65: 612-626

26 Fiore R, Khudayberdiev S, Christensen M, et al. Mef2-mediated transcription of the miR379-410 cluster regulates activity-dependent dendritogenesis by fine-tuning Pumilio2 protein levels. EMBO J, 2009, 28: 697-710

27 Kye M J, Liu T, Levy S F, et al. Somatodendritic microRNAs identified by laser capture and multiplex RT-PCR. RNA, 2007, 13: 1224-1234

28 Lugli G, Torvik V I, Larson J, et al. Expression of microRNAs and their precursors in synaptic fractions of adult mouse forebrain. $\mathrm{J}$ Neurochem, 2008, 106: 650-661

29 Bernstein E, Kim S Y, Carmell M A, et al. Dicer is essential for mouse development. Nat Genet, 2003, 35: 215-217

30 De Pietri T D, Pulvers J N, Haffner C, et al. miRNAs are essential for survival and differentiation of newborn neurons but not for expansion of neural progenitors during early neurogenesis in the mouse embryonic neocortex. Development, 2008, 135: 3911-3921

31 Kawase-Koga Y, Otaegi G, Sun T. Different timings of Dicer dele- tion affect neurogenesis and gliogenesis in the developing mouse central nervous system. Dev Dyn, 2009, 238: 2800-2812

32 Kim J, Inoue K, Ishii J, et al. A microRNA feedback circuit in midbrain dopamine neurons. Science, 2007, 317: 1220-1224

33 Schaefer A, O'Carroll D, Tan C L, et al. Cerebellar neurodegeneration in the absence of microRNAs. J Exp Med, 2007, 204: 1553-1558

34 Davis T H, Cuellar T L, Koch S M, et al. Conditional loss of Dicer disrupts cellular and tissue morphogenesis in the cortex and hippocampus. J Neurosci, 2008, 28: 4322-4330

35 Stark K L, Xu B, Bagchi A, et al. Altered brain microRNA biogenesis contributes to phenotypic deficits in a 22q11-deletion mouse model. Nat Genet, 2008, 40: 751-760

36 Fenelon K, Mukai J, Xu B, et al. Deficiency of Dgcr8, a gene disrupted by the 22q11.2 microdeletion, results in altered short-term plasticity in the prefrontal cortex. Proc Natl Acad Sci USA, 2011, 108: 4447-4452

37 Schofield C M, Hsu R, Barker A J, et al. Monoallelic deletion of the microRNA biogenesis gene Dgcr8 produces deficits in the development of excitatory synaptic transmission in the prefrontal cortex. Neural Dev, 2011, 6: 11

38 Baumann N, Pham-Dinh D. Biology of oligodendrocyte and myelin in the mammalian central nervous system. Physiol Rev, 2001, 81: 871-927

39 Shin D, Shin J Y, McManus M T, et al. Dicer ablation in oligodendrocytes provokes neuronal impairment in mice. Ann Neurol, 2009, 66: 843-857

40 Pereira J A, Baumann R, Norrmen C, et al. Dicer in Schwann cells is required for myelination and axonal integrity. J Neurosci, 2010, 30: 6763-6775

41 Yun B, Anderegg A, Menichella D, et al. MicroRNA-deficient Schwann cells display congenital hypomyelination. J Neurosci, 2010, 30: $7722-7728$

42 Tao J, Wu H, Lin Q, et al. Deletion of astroglial Dicer causes non-cell-autonomous neuronal dysfunction and degeneration. J Neurosci, 2011, 31: 8306-8319

43 Makeyev E V, Zhang J, Carrasco M A, et al. The MicroRNA miR124 promotes neuronal differentiation by triggering brain-specific alternative pre-mRNA splicing. Mol Cell, 2007, 27: 435-448

44 Yoo A S, Staahl B T, Chen L, et al. MicroRNA-mediated switching of chromatin-remodelling complexes in neural development. Nature, 2009, 460: 642-646

45 Yoo A S, Sun A X, Li L, et al. MicroRNA-mediated conversion of human fibroblasts to neurons. Nature, 2011, 476: 228-231

46 Lugli G, Larson J, Martone M E, et al. Dicer and eIF2c are enriched at postsynaptic densities in adult mouse brain and are modified by neuronal activity in a calpain-dependent manner. J Neurochem, 2005, 94: 896-905

47 Siegel G, Obernosterer G, Fiore R, et al. A functional screen implicates microRNA-138-dependent regulation of the depalmitoylation enzyme APT1 in dendritic spine morphogenesis. Nat Cell Biol, 2009, 11: 705-716

48 Cuellar T L, Davis T H, Nelson P T, et al. Dicer loss in striatal neurons produces behavioral and neuroanatomical phenotypes in the absence of neurodegeneration. Proc Natl Acad Sci USA, 2008, 105: 5614-5619

49 Haramati S, Chapnik E, Sztainberg Y, et al. miRNA malfunction causes spinal motor neuron disease. Proc Natl Acad Sci USA, 2010, 107: 13111-13116

50 Kawase-Koga Y, Low R, Otaegi G, et al. RNAase-III enzyme Dicer maintains signaling pathways for differentiation and survival in mouse cortical neural stem cells. J Cell Sci, 2010, 123: 586-594

51 Hebert S S, Papadopoulou A S, Smith P, et al. Genetic ablation of Dicer in adult forebrain neurons results in abnormal tau hyperphosphorylation and neurodegeneration. Hum Mol Genet, 2010, 19: 3959-3969

52 Konopka W, Kiryk A, Novak M, et al. MicroRNA loss enhances learning and memory in mice. J Neurosci, 2010, 30: 14835-14842

53 Damiani D, Alexander J J, O'Rourke J R, et al. Dicer inactivation leads to progressive functional and structural degeneration of the 
mouse retina. J Neurosci, 2008, 28: 4878-4887

54 Georgi S A, Reh T A. Dicer is required for the transition from early to late progenitor state in the developing mouse retina. J Neurosci, 2010, 30: 4048-4061

55 Iida A, Shinoe T, Baba Y, et al. Dicer plays essential roles for retinal development by regulation of survival and differentiation. Invest Ophthalmol Vis Sci, 2011, 52: 3008-3017

56 Friedman L M, Dror A A, Mor E, et al. MicroRNAs are essential for development and function of inner ear hair cells in vertebrates. Proc Natl Acad Sci USA, 2009, 106: 7915-7920

57 Choi P S, Zakhary L, Choi W Y, et al. Members of the miRNA-200 family regulate olfactory neurogenesis. Neuron, 2008, 57: 41-55

58 Zheng K, Li H, Zhu Y, et al. MicroRNAs are essential for the developmental switch from neurogenesis to gliogenesis in the developing spinal cord. J Neurosci, 2010, 30: 8245-8250

59 Budde H, Schmitt S, Fitzner D, et al. Control of oligodendroglial cell number by the miR-17-92 cluster. Development, 2010, 137: 2127-2132

60 Vo N, Klein M E, Varlamova O, et al. A cAMP-response element binding protein-induced microRNA regulates neuronal morphogenesis. Proc Natl Acad Sci USA, 2005, 102: 16426-16431

61 Obernosterer G, Leuschner P J, Alenius M, et al. Post-transcriptional regulation of microRNA expression. RNA, 2006, 12: 1161-1167

62 Kedde M, Agami R. Interplay between microRNAs and RNAbinding proteins determines developmental processes. Cell Cycle, 2008, 7: 899-903

63 Muddashetty R S, Nalavadi V C, Gross C, et al. Reversible inhibition of PSD-95 mRNA translation by miR-125a, FMRP phosphorylation, and mGluR signaling. Mol Cell, 2011, 42: 673-688

64 Wayman G A, Davare M, Ando H, et al. An activity-regulated microRNA controls dendritic plasticity by down-regulating p250GAP. Proc Natl Acad Sci USA, 2008, 105: 9093-9098

65 Schratt G M, Tuebing F, Nigh E A, et al. A brain-specific microRNA regulates dendritic spine development. Nature, 2006, 439: 283-289

66 Lau P, Verrier J D, Nielsen J A, et al. Identification of dynamically regulated microRNA and mRNA networks in developing oligodendrocytes. J Neurosci, 2008, 28: 11720-11730

67 Hart I K, Richardson W D, Raff M C. PDGF increases the expression of Fos and Jun in newly formed oligodendrocytes that have become resistant to the mitogenic effect of PDGF. Glia, 1992, 6: 310-313

68 Kleihues P, Louis D N, Scheithauer B W. World Health Organization Classification of Tumours of the Nervous System. 2000

69 Roussel M F, Hatten M E. Cerebellum development and medulloblastoma. Curr Top Dev Biol, 2011, 94: 235-282

70 Turner J D, Williamson R, Almefty K K, et al. The many roles of microRNAs in brain tumor biology. Neurosurg Focus, 2010, 28: E3

71 Chen Y, Liu W, Chao T, et al. MicroRNA-21 down-regulates the expression of tumor suppressor PDCD4 in human glioblastoma cell T98G. Cancer Lett, 2008, 272: 197-205

72 Papagiannakopoulos T, Shapiro A, Kosik K S. MicroRNA-21 targets a network of key tumor-suppressive pathways in glioblastoma cells. Cancer Res, 2008, 68: 8164-8172

73 Gabriely G, Wurdinger T, Kesari S, et al. MicroRNA 21 promotes glioma invasion by targeting matrix metalloproteinase regulators. Mol Cell Biol, 2008, 28: 5369-5380

74 Li Y, Li W, Yang Y, et al. MicroRNA-21 targets LRRFIP1 and contributes to VM-26 resistance in glioblastoma multiforme. Brain Res, 2009, 1286: 13-18

75 Kwak H J, Kim Y J, Chun K R, et al. Downregulation of Spry2 by miR-21 triggers malignancy in human gliomas. Oncogene, 2011, 30: 2433-2442

76 Grunder E, D’Ambrosio R, Fiaschetti G, et al. MicroRNA-21 suppression impedes medulloblastoma cell migration. Eur $\mathrm{J}$ Cancer, 2011, in press

77 Zhang C Z, Zhang J X, Zhang A L, et al. MiR-221 and miR-222 target PUMA to induce cell survival in glioblastoma. Mol Cancer, 2010, 9: 229

78 Quintavalle C, Garofalo M, Zanca C, et al. miR-221/222 overexpression in human glioblastoma increases invasiveness by targeting the protein phosphate PTPmu. Oncogene, 2011, in press

79 Gillies J K, Lorimer I A. Regulation of p27Kip1 by miRNA 221/222 in glioblastoma. Cell Cycle, 2007, 6: 2005-2009

80 Ciafre S A, Galardi S, Mangiola A, et al. Extensive modulation of a set of microRNAs in primary glioblastoma. Biochem Biophys Res Commun, 2005, 334: 1351-1358

81 Lukiw W J, Cui J G, Li Y Y, et al. Up-regulation of micro-RNA-221 (miRNA-221; chr Xp11.3) and caspase-3 accompanies down-regulation of the survivin-1 homolog BIRC1 (NAIP) in glioblastoma multiforme (GBM). J Neurooncol, 2009, 91: 27-32

82 Ernst A, Campos B, Meier J, et al. De-repression of CTGF via the miR-17-92 cluster upon differentiation of human glioblastoma spheroid cultures. Oncogene, 2010, 29: 3411-3422

83 Uziel T, Karginov F V, Xie S, et al. The miR-17 92 cluster collaborates with the Sonic Hedgehog pathway in medulloblastoma. Proc Natl Acad Sci USA, 2009, 106: 2812-2817

84 Northcott P A, Fernandez-L A, Hagan J P, et al. The miR-17/92 polycistron is up-regulated in sonic hedgehog-driven medulloblastomas and induced by $\mathrm{N}$-myc in sonic hedgehog-treated cerebellar neural precursors. Cancer Res, 2009, 69: 3249-3255

85 Sasayama T, Nishihara M, Kondoh T, et al. MicroRNA-10b is overexpressed in malignant glioma and associated with tumor invasive factors, uPAR and RhoC. Int J Cancer, 2009, 125: 1407-1413

86 Sun L, Yan W, Wang Y, et al. MicroRNA-10b induces glioma cell invasion by modulating MMP-14 and uPAR expression via HOXD10. Brain Res, 2011, 1389: 9-18

87 Gabriely G, Yi M, Narayan R S, et al. Human glioma growth is controlled by microRNA-10b. Cancer Res, 2011, 71: 3563-3572

88 Huse J T, Brennan C, Hambardzumyan D, et al. The PTEN-regulating microRNA miR-26a is amplified in high-grade glioma and facilitates gliomagenesis in vivo. Genes Dev, 2009, 23: 1327-1337

89 Kim H, Huang W, Jiang X, et al. Integrative genome analysis reveals an oncomir/oncogene cluster regulating glioblastoma survivorship. Proc Natl Acad Sci USA, 2010, 107: 2183-2188

90 Weeraratne S D, Amani V, Neiss A, et al. miR-34a confers chemosensitivity through modulation of MAGE-A and p53 in medulloblastoma. Neuro Oncol, 2011, 13: 165-175

91 Luan S, Sun L, Huang F. MicroRNA-34a: a novel tumor suppressor in p53-mutant glioma cell line U251. Arch Med Res, 2010, 41: 67-74

92 Li Y, Guessous F, Zhang Y, et al. MicroRNA-34a inhibits glioblastoma growth by targeting multiple oncogenes. Cancer Res, 2009, 69: 7569-7576

93 Guessous F, Zhang Y, Kofman A, et al. microRNA-34a is tumor suppressive in brain tumors and glioma stem cells. Cell Cycle, 2010, 9: $1031-1036$

94 Yang G, Zhang R, Chen X, et al. MiR-106a inhibits glioma cell growth by targeting E2F1 independent of p53 status. J Mol Med (Berl), 2011, 89: 1037-1050

95 Pierson J, Hostager B, Fan R, et al. Regulation of cyclin dependent kinase 6 by microRNA 124 in medulloblastoma. J Neurooncol, 2008, 90: $1-7$

96 Li K K, Pang J C, Ching A K, et al. miR-124 is frequently down-regulated in medulloblastoma and is a negative regulator of SLC16A1. Hum Pathol, 2009, 40: 1234-1243

97 Silber J, Lim D A, Petritsch C, et al. miR-124 and miR-137 inhibit proliferation of glioblastoma multiforme cells and induce differentiation of brain tumor stem cells. BMC Med, 2008, 6: 14

98 Zhang Y, Chao T, Li R, et al. MicroRNA-128 inhibits glioma cells proliferation by targeting transcription factor E2F3a. J Mol Med (Berl), 2009, 87: 43-51

99 Cui J G, Zhao Y, Sethi P, et al. Micro-RNA-128 (miRNA-128) down-regulation in glioblastoma targets ARP5 (ANGPTL6), Bmi-1 and E2F-3a, key regulators of brain cell proliferation. J Neurooncol, 2010, 98: 297-304

100 Venkataraman S, Alimova I, Fan R, et al. MicroRNA 128a increases intracellular ROS level by targeting Bmi-1 and inhibits medulloblastoma cancer cell growth by promoting senescence. PLoS ONE, 2010, 5: e10748 
101 Xia H F, He T Z, Liu C M, et al. MiR-125b expression affects the proliferation and apoptosis of human glioma cells by targeting Bmf. Cell Physiol Biochem, 2009, 23: 347-358

102 Shi L, Zhang J, Pan T, et al. MiR-125b is critical for the suppression of human U251 glioma stem cell proliferation. Brain Res, 2010, 1312: $120-126$

103 Garzia L, Andolfo I, Cusanelli E, et al. MicroRNA-199b-5p impairs cancer stem cells through negative regulation of HES1 in medulloblastoma. PLoS ONE, 2009, 4: e4998

104 Ciafre S A, Galardi S, Mangiola A, et al. Extensive modulation of a set of microRNAs in primary glioblastoma. Biochem Biophys Res Commun, 2005, 334: 1351-1358

105 Ferretti E, De Smaele E, Po A, et al. MicroRNA profiling in human medulloblastoma. Int J Cancer, 2009, 124: 568-577

106 Corsten M F, Miranda R, Kasmieh R, et al. MicroRNA-21 knockdown disrupts glioma growth in vivo and displays synergistic cytotoxicity with neural precursor cell delivered S-TRAIL in human gliomas. Cancer Res, 2007, 67: 8994-9000

107 Zhou X, Ren Y, Moore L, et al. Downregulation of miR-21 inhibits EGFR pathway and suppresses the growth of human glioblastoma cells independent of PTEN status. Lab Invest, 2010, 90: 144-155

108 Ren Y, Zhou X, Mei M, et al. MicroRNA-21 inhibitor sensitizes human glioblastoma cells U251 (PTEN-mutant) and LN229 (PTEN-wild type) to taxol. BMC Cancer, 2010, 10: 27

109 Zhou X, Zhang J, Jia Q, et al. Reduction of miR-21 induces glioma cell apoptosis via activating caspase 9 and 3. Oncol Rep, 2010, 24: 195-201

110 Fineberg S K, Kosik K S, Davidson B L. MicroRNAs potentiate neural development. Neuron, 2009, 64: 303-309

111 Jin P, Zarnescu D C, Ceman S, et al. Biochemical and genetic interaction between the fragile $\mathrm{X}$ mental retardation protein and the microRNA pathway. Nat Neurosci, 2004, 7: 113-117

112 Edbauer D, Neilson J R, Foster K A, et al. Regulation of synaptic structure and function by FMRP-associated microRNAs miR-125b and miR-132. Neuron, 2010, 65: 373-384

$113 \mathrm{Xu} \mathrm{X} \mathrm{L,} \mathrm{Li} \mathrm{Y,} \mathrm{Wang} \mathrm{F,} \mathrm{et} \mathrm{al.} \mathrm{The} \mathrm{steady-state} \mathrm{level} \mathrm{of} \mathrm{the} \mathrm{nerv-}$ ous-system-specific microRNA-124a is regulated by dFMR1 in Drosophila. J Neurosci, 2008, 28: 11883-11889

114 Samaco R C, Neul J L. Complexities of Rett syndrome and MeCP2. J Neurosci, 2011, 31: 7951-7959

115 Klein M E, Lioy D T, Ma L, et al. Homeostatic regulation of MeCP2 expression by a CREB-induced microRNA. Nat Neurosci, 2007, 10: 1513-1514
116 Urdinguio R G, Fernandez A F, Lopez-Nieva P, et al. Disrupted microRNA expression caused by Mecp2 loss in a mouse model of Rett syndrome. Epigenetics, 2010, 5: 656-663

$117 \mathrm{Wu} \mathrm{H}$, Tao J, Chen $\mathrm{P}$ J, et al. Genome-wide analysis reveals methyl-CpG-binding protein 2-dependent regulation of microRNAs in a mouse model of Rett syndrome. Proc Natl Acad Sci USA, 2010, 107: 18161-18166

118 Lees A J, Hardy J, Revesz T. Parkinson's disease. Lancet, 2009, 373 : 2055-2066

119 Junn E, Lee K W, Jeong B S, et al. Repression of alpha-synuclein expression and toxicity by microRNA-7. Proc Natl Acad Sci USA, 2009, 106: 13052-13057

120 Doxakis E. Post-transcriptional regulation of alpha-synuclein expression by mir-7 and mir-153. J Biol Chem, 2010, 285: 12726-12734

121 Cogswell J P, Ward J, Taylor I A, et al. Identification of miRNA changes in Alzheimer's disease brain and CSF yields putative biomarkers and insights into disease pathways. J Alzheimers Dis, 2008, 14: 27-41

122 Wang W X, Rajeev B W, Stromberg A J, et al. The expression of microRNA miR-107 decreases early in Alzheimer's disease and may accelerate disease progression through regulation of beta-site amyloid precursor protein-cleaving enzyme 1. J Neurosci, 2008, 28: 1213-1223

123 Hebert S S, Horre K, Nicolai L, et al. Loss of microRNA cluster miR-29a/b-1 in sporadic Alzheimer's disease correlates with increased BACE1/beta-secretase expression. Proc Natl Acad Sci USA, 2008, 105: 6415-6420

124 Boissonneault V, Plante I, Rivest S, et al. MicroRNA-298 and microRNA-328 regulate expression of mouse beta-amyloid precursor protein-converting enzyme 1. J Biol Chem, 2009, 284: 1971-1981

125 Hebert S S, Horre K, Nicolai L, et al. MicroRNA regulation of Alzheimer's Amyloid precursor protein expression. Neurobiol Dis, 2009, 33: 422-428

126 Vilardo E, Barbato C, Ciotti M, et al. MicroRNA-101 regulates amyloid precursor protein expression in hippocampal neurons. J Biol Chem, 2010, 285: 18344-18351

127 Liu W, Liu C, Zhu J, et al. MicroRNA-16 targets amyloid precursor protein to potentially modulate Alzheimer's-associated pathogenesis in SAMP8 mice. Neurobiol Aging, 2010

128 Long J M, Lahiri D K. MicroRNA-101 downregulates Alzheimer's amyloid-beta precursor protein levels in human cell cultures and is differentially expressed. Biochem Biophys Res Commun, 2011, 404: 889-895

Open Access This article is distributed under the terms of the Creative Commons Attribution License which permits any use, distribution, and reproduction in any medium, provided the original author(s) and source are credited. 